\title{
On a General Class of $q$-Rational Type Operators
}

\author{
NURHAYAT İSPIR
}

\begin{abstract}
In this study, we define a general class of rational type operators based on $q$-calculus and investigate the weighted approximation properties of these operators by using $A$-statistical convergence. We also estimate the rates of $A$-statistical convergence of these operators by modulus of continuity and Petree's $K$-functional. The operators to be introduced, include some well known $q$-operators so our results are true in a large spectrum of these operators.
\end{abstract}

\section{INTRODUCTION}

K. Balazs [3] introduced the Bernstein type rational functions and proved the convergence theorems for them. Later, K.Balazs and J.Szabados [4] improved some estimates on the order of approximation for the Bernstein type rational operators.

The generalization of the Bernstein type rational operators are introduced by C. Atakut and N. Ispir [16] as follows

$$
L_{n}(f)(x)=\frac{1}{\psi_{n}\left(a_{n} x\right)} \sum_{k=0}^{\infty} f\left(\frac{k}{b_{n}}\right) \frac{\psi_{n}^{(k)}(0)}{k !}\left(a_{n} x\right)^{k}, \quad n \in \mathbb{N}, x \geq 0,
$$

where $a_{n}$ and $b_{n}$ are suitably chosen real numbers, independent of $x$. Here $\left\{\psi_{n}\right\}$ is a sequence of functions $\psi_{n}: \mathbb{C} \rightarrow \mathbb{C}$ satisfying the following conditions:

a) $\psi_{n}(n=1,2, \ldots)$ is analytic on a domain $D$ containing the disk

$$
B=\{z \in \mathbb{C}:|z-b| \leq b\}
$$

b) $\psi_{n}(0)=1, \quad(n=1,2, \ldots)$;

c) For any $x \geq 0, \psi_{n}(x)>0$ and $\psi_{n}^{(k)}(0) \geq 0$ for any $n=1,2, \ldots$, $k=1,2, \ldots$

2010 Mathematics Subject Classification. Primary: 41A25, 41A36, 40 A35.

Key words and phrases. $q$-calculus, rational type operators, $A$-statistical convergence, weighted spaces, linear positive operators. 
d) For every $n=1,2, \ldots$, it is

$$
\lim _{n}\left(\frac{\psi_{n}^{(\nu)}\left(a_{n} x\right)}{n^{\nu} \psi_{n}\left(a_{n} x\right)}-1\right)=0 ; \quad \nu=1,2,
$$

where $a_{n} \rightarrow 0$, as $n \rightarrow \infty$.

In [16] the authors estimated the order of approximation for the operators defined by (1) and proved a Voronovskaja type asymptotic formula and pointwise convergence in simultaneous approximation. In [13, 14, 18] the approximation properties for different variants of the operators (1) were investigated in various function spaces . In [17], the approximation properties of the Kantorovich variant of the operators (1) were given by the aid of $A$-statistical convergence. Notice that $A$-statistical convergence is stronger than usual convergence.

It is known that the applications of $q$-calculus in the area of approximation theory have been an active area of research. In the recent years, the statistical approximation properties of some positive linear operators based on $q$-integers have been studied intensively by many authors (e.g. $[5,15,17,21])$.

The aim of this study is to introduce $q$-type generalization of the operators (1) and investigate the $A$-statistical approximation properties of the constructed operators in weighted spaces. Using $A$-statistical convergence, we obtain weighted Korovkin type theorem and weighted order of approximation by the constructed operators based $q$-calculus. Moreover, we estimate the rate of $A$-statistical convergence by usual modulus of continuity and by Petree's $K$-functional in the different normed spaces for $q$-extension of the operators (1).

Now, let us give a few basic definitions and notations in $q$-integers shortly. Details on $q$-calculus can be found in [7]. Throughout the present paper, we consider $q$ as a real number such that $0<q<1$, and for each nonnegative integer $i$, the $q$-integer $[i]_{q}$ is defined by $[i]_{q}=\left(1-q^{i}\right) /(1-q),[0]_{q}:=0$; and $q$-factorial $[i]_{q}$ ! is defined by $[i]_{q} !=[1]_{q}[2]_{q} \ldots[i]_{q},[0] !:=1$.

For fixed $0<q<1$, the $q$-derivative of a function $f: \mathbb{R} \rightarrow \mathbb{R}$ with respect to $x$ is defined by $D_{q} f(x)=\frac{f(q x)-f(x)}{(q-1) x}, x \neq 0$ and $D_{q} f(0)=\lim _{x \rightarrow 0} D_{q} f(x)$. The chain rule for ordinary derivatives is similar for $q$-derivative.

At this point, we recall the $q$-Taylor theorem in the following. Theorem $\mathbf{A}([7]$, p. 103.) If a function $f(x)$ possess convergence series expansion then

$$
f(x)=\sum_{n=0}^{\infty} \frac{(x-a)_{q}^{n}}{[n]_{q} !} D_{q}^{n}[f(a)]
$$

where $(x-a)_{q}^{n}=\prod_{s=0}^{n-1}\left(x-q^{s} a\right)=\sum_{k=0}^{n}\left[\begin{array}{l}n \\ k\end{array}\right]_{q} q^{k(k-1)} x^{n-k}(-a)^{k}$.

Now let us recall some concepts of the $A$-statistical convergence. Suppose that $A$ is non-negative summability matrix and let $K$ be subset of $\mathbb{N}$ 
the set of natural numbers. The $A$-density of $K$ is defined by $\delta_{A}(K):=$ $\lim _{j} \frac{1}{n} \sum_{n=1}^{\infty} a_{j n} \chi_{K}(n)$ provided limit exists, where $\chi_{K}$ characteristic function of $K$. A sequence $x=\left(x_{n}\right)$ is called $A$-statistically convergent to $L$ if for every $\varepsilon>0 \lim _{j} \sum_{n:\left|x_{n}-L\right| \geq \varepsilon} a_{j n}=0$ or equivalently for every $\varepsilon>0$, $\delta_{A}\left\{k \in \mathbb{N}:\left|x_{k}-L\right| \geq \varepsilon\right\}=0$. In this case we write $s t_{A}-\lim x=L[8,9]$.

The case in which $A=C_{1}$, the Cesáro matrix of order one, $A$-statistical convergence reduces to the statistical convergence [9, 19]. Also if $A=I$, the identity matrix, then it reduces to the ordinary convergence. We note that, if $A=\left(a_{j n}\right)$ is a non-negative regular matrix such that $\lim _{j} \max _{n}\left\{a_{j n}\right\}=0$, then A-statistical convergence is stronger than convergence [19]. It should be noted that the concept of $A$-statistical convergence may also be given in normed spaces: Assume $(X,\|\cdot\|)$ is a normed space and $u=\left(u_{k}\right)$ is a $X$-valued sequence. Then $\left(u_{k}\right)$ is said to be A-statistically convergent

to $u_{0} \in X$ if, for every $\varepsilon>0, \delta_{A}\left\{k \in \mathbb{N}:\left\|u_{k}-u_{0}\right\| \geq \varepsilon\right\}=0$ [19].

\section{CONSTRUCTION OF OPERATORS AND AUXILIARY RESULTS}

Now we would like to introduce $q$-generalization of the operators (1).

Let $\left(\varphi_{n}\right)$ be a sequence of real functions on $\mathbb{R}_{+}$which are continuously infinitely $q$-differentiable on $\mathbb{R}_{+}$satisfying the following conditions

1. $\varphi_{n}(0)=1$, for each $n \in \mathbb{N}$

2. $D_{q}^{k} \varphi_{n}(0) \geq 0$, for every $n, k \in \mathbb{N}, x \geq 0$

3. For every $n \in \mathbb{N}$, with $a_{n}=[n]_{q}^{\beta-1}, b_{n}=[n]_{q}^{\beta}, q \in(0,1], 0<\beta \leq \frac{2}{3}$

$$
s t_{A}-\lim _{n}\left(\frac{D_{q}^{\nu} \varphi_{n}\left([n]_{q}^{\beta-1} x\right)}{q^{\nu-1}[n]_{q}^{\nu} \varphi_{n}\left([n]_{q}^{\beta-1} x\right)}-1\right)=0 ; \quad \nu=1,2 .
$$

For fixed $x \in \mathbb{R}_{+}$, taking account to Theorem $\mathrm{A}$ we get

$$
\varphi_{n}\left([n]_{q}^{\beta-1} x\right)=\sum_{k=0}^{\infty} \frac{\left([n]_{q}^{\beta-1} x\right)_{q}^{k}}{[k]_{q} !} D_{q}^{k} \varphi_{n}(0) .
$$

Using the formula $(a+b)_{q}^{n}=\prod_{s=0}^{n-1}\left(a+q^{s} b\right)=\sum_{k=0}^{n} q^{k(k-1) / 2}\left[\begin{array}{l}n \\ k\end{array}\right]_{q} b^{k} a^{n-k}$ and taking $a=0, b=[n]_{q}^{\beta-1} x$ we write $\left([n]_{q}^{\beta-1} x\right)_{q}^{k}=q^{k(k-1) / 2}\left([n]_{q}^{\beta-1} x\right)^{k}$. Choosing $a_{n}=[n]_{q}^{\beta-1}, b_{n}=[n]_{q}^{\beta}, 0<\beta \leq \frac{2}{3}, n \in \mathbb{N}$, we introduce $q$ generalization of the operator (1) as follows

$$
L_{n, q}(f)(x)=\frac{1}{\varphi_{n}\left([n]_{q}^{\beta-1} x\right)} \sum_{k=0}^{\infty} f\left(\frac{[k]_{q}}{[n]_{q}^{\beta} q^{k-1}}\right) q^{k(k-1) / 2} \frac{\left([n]_{q}^{\beta-1} x\right)^{k}}{[k]_{q} !} D_{q}^{k} \varphi_{n}(0),
$$


for each $n \in \mathbb{N}, \quad x \geq 0$.

Note that:

- It is easily verified that the operators $L_{n, q}$ are linear positive operators.

- The order of convergence is the best possible estimate for $\beta \in(0,2 / 3]$ (see [4]).

- The present condition (3) is weaker than the present one given by (2) for $q=1$. Indeed, we can construct a sequence such that it is statistically convergent to 1 but not convergent in the ordinary sense. A well known example is defined as; $\alpha_{n}=\sqrt{n}$ if $n=m^{2}(m \in \mathbf{N})$, and $\alpha_{n}=1$ otherwise. Same result also works to $A$-statistical convergence.

Lemma 2.1. For all $n \in \mathbb{N}, x \geq 0$ and $0<q<1$ we get

$$
L_{n, q}\left(e_{0}\right)(x)=1 \text {, }
$$

$$
L_{n, q}\left(e_{1}\right)(x)=\frac{D_{q} \varphi_{n}\left([n]_{q}^{\beta-1} x\right)}{[n]_{q} \varphi_{n}\left([n]_{q}^{\beta-1} x\right)} x
$$

(8) $\quad L_{n, q}\left(e_{2}\right)(x)=\frac{D_{q}^{2} \varphi_{n}\left([n]_{q}^{\beta-1} x\right)}{q[n]_{q}^{2} \varphi_{n}\left([n]_{q}^{\beta-1} x\right)} x^{2}+\frac{1}{[n]_{q}^{\beta}} \frac{D_{q} \varphi_{n}\left([n]_{q}^{\beta-1} x\right)}{[n]_{q} \varphi_{n}\left([n]_{q}^{\beta-1} x\right)} x$

where $\left(e_{i}\right)(x)=x^{i}, i=0,1,2$.

Proof. From (4) and definition of $L_{n, q}(f)$ it is clear that $L_{n, q}\left(e_{0}\right)(x)=1$. Considering (4), we can write the $q$-derivative of $\varphi_{n}$ with respect to $x$ as

$$
\begin{gathered}
{[n]_{q}^{\beta-1} D_{q} \varphi_{n}\left([n]_{q}^{\beta-1} x\right)=\sum_{k=1}^{\infty} \frac{[k]_{q}}{[k]_{q} !}[n]_{q}^{\beta-1}\left([n]_{q}^{\beta-1} x\right)_{q}^{k-1} D_{q}^{k} \varphi_{n}(0)} \\
=\sum_{k=1}^{\infty} \frac{[k]_{q}}{[k]_{q} !}[n]_{q}^{\beta-1} q^{(k-1)(k-2) / 2}\left([n]_{q}^{\beta-1} x\right)^{k-1} D_{q}^{k} \varphi_{n}(0)
\end{gathered}
$$

where used the equation $\left(a_{n} x\right)_{q}^{k}=q^{k(k-1)}\left(a_{n} x\right)^{k}$. Hence multiplying both sides by $x$ and dividing by $[n]_{q}^{\beta} \varphi_{n}\left([n]_{q}^{\beta-1} x\right)$ we obtain

$$
\begin{aligned}
& \frac{D_{q} \varphi_{n}\left([n]_{q}^{\beta-1} x\right)}{[n]_{q} \varphi_{n}\left([n]_{q}^{\beta-1} x\right)} x= \\
& \quad=\frac{1}{\varphi_{n}\left([n]_{q}^{\beta-1} x\right)} \sum_{k=1}^{\infty} \frac{[k]_{q}}{[n]_{q}^{\beta} q^{k-1}} q^{k(k-1) / 2} \frac{\left([n]_{q}^{\beta-1} x\right)^{k}}{[k]_{q} !} D_{q}^{k} \varphi_{n}(0)
\end{aligned}
$$


which gives the (7). We use a similar technique to get (8). Again differentiating (9) with respect to $x$ we have

$$
\begin{aligned}
& \left([n]_{q}^{\beta-1}\right)^{2} D_{q}^{2} \varphi_{n}\left([n]_{q}^{\beta-1} x\right) \\
& \quad=\sum_{k=2}^{\infty} \frac{[k]_{q}[k-1]_{q}}{[k]_{q} !}\left([n]_{q}^{\beta-1}\right)^{2} q^{(k-2)(k-3) / 2}\left([n]_{q}^{\beta-1} x\right)^{k-2} D_{q}^{k} \varphi_{n}(0) .
\end{aligned}
$$

Using the equality $[k-1]_{q}=\left([k]_{q}-q^{k-1}\right)$ and multiplying both sides by $x^{2}$ we have

$$
\begin{aligned}
& \left([n]_{q}^{\beta-1} x\right)^{2} D_{q}^{2} \varphi_{n}\left([n]_{q}^{\beta-1} x\right) \\
& =\sum_{k=1}^{\infty} \frac{[k]_{q}^{2}}{[k]_{q} !} q^{(k-2)(k-3) / 2}\left([n]_{q}^{\beta-1} x\right)^{k} D_{q}^{k} \varphi_{n}(0) \\
& \quad-\sum_{k=1}^{\infty} \frac{[k]_{q}}{[k]_{q} !} q^{k-1} q^{(k-1)(k-2) / 2}\left([n]_{q}^{\beta-1} x\right)^{k} D_{q}^{k} \varphi_{n}(0) \\
& =q \sum_{k=1}^{\infty}\left(\frac{[k]_{q}}{q^{k-1}}\right)^{2} q^{k(k-1) / 2}\left([n]_{q}^{\beta-1} x\right)^{k} \frac{D_{q}^{k} \varphi_{n}(0)}{[k]_{q} !} \\
& \quad-q \sum_{k=1}^{\infty} \frac{[k]_{q}}{q^{k-1}} q^{k(k-1) / 2}\left([n]_{q}^{\beta-1} x\right)^{k} \frac{D_{q}^{k} \varphi_{n}(0)}{[k]_{q} !} .
\end{aligned}
$$

Dividing by $[n]_{q}^{2 \beta} \varphi_{n}\left([n]_{q}^{\beta-1} x\right)$ we write

$$
\begin{aligned}
& \frac{\left([n]_{q}^{\beta-1} x\right)^{2} D_{q}^{2} \varphi_{n}\left([n]_{q}^{\beta-1} x\right)}{[n]_{q}^{\beta \beta} \varphi_{n}\left([n]_{q}^{\beta-1} x\right)} \\
= & \frac{q}{\varphi_{n}\left([n]_{q}^{\beta-1} x\right)} \sum_{k=1}^{\infty}\left(\frac{[k]_{q}}{[n]_{q}^{\beta} q^{k-1}}\right)^{2} q^{k(k-1) / 2}\left([n]_{q}^{\beta-1} x\right)^{k} \frac{D_{q}^{k} \varphi_{n}(0)}{[k]_{q} !} \\
& -\frac{q}{[n]_{q}^{\beta} \varphi_{n}\left([n]_{q}^{\beta-1} x\right)} \sum_{k=1}^{\infty} \frac{[k]_{q}}{[n]_{q}^{\beta} q^{k-1}} q^{k(k-1) / 2}\left([n]_{q}^{\beta-1} x\right)^{k} \frac{D_{q}^{k} \varphi_{n}(0)}{[k]_{q} !}
\end{aligned}
$$

which gives the (8) by using formulas (5) and (10).

\section{A-Statistical CONVERGenCe in Weighted SPACES}

Let $\rho$ denotes a continuous weight function with $\rho(x) \geq 1, x \in[0, \infty)$ and $\rho(x) \rightarrow \infty$ as $x \rightarrow \infty$. Let $B_{\rho}$ be the weighted space of all functions $f$ defined on the $\mathbb{R}_{+}$satisfying the condition $|f(x)| \leq M_{f} \rho(x)$ with some constant $M_{f}$, depending only on $f$. By $C_{\rho}$, let us denote the subspace of 
all continuous functions belong to $B_{\rho}$. Also, let $C_{\rho}^{0}$ be the subspace of all functions $f \in C_{\rho}$ for which $\lim _{|x| \rightarrow \infty} f(x) / \rho(x)=0$. Endowed with the norm $\|f\|_{\rho}=\sup _{x \geq 0}(|f(x)| / \rho(x))$ these spaces are Banach spaces. Note that the weighted Korovkin type theorem were proved by A.D. Gadjiev [10, 11]. Using $A$-statistical convergence, the weighted Korovkin type theorem was given in [6].

Let $\left\{L_{n, q}\right\}$ be the sequence of linear positive operators defined by (5). Then it is easily seen that $L_{n, q}: C_{\rho} \rightarrow B_{\rho}$.

Let $q=\left\{q_{n}\right\}$ be a sequence satisfying the following conditions

$$
s t_{A}-\lim _{n} q_{n}=1 \quad \text { and } \quad s t_{A}-\lim _{n} q_{n}^{n}=a, \quad(0 \leq a<1) .
$$

The condition (12) guaranties that $s t_{A}-\lim _{n}\left([n]_{q}^{-1}\right)=0$.

Now we are ready to prove our first result which is related to the $A$ statistical convergence the sequence of $\left\{L_{n, q_{n}}(f)\right\}$ to $f$.

Theorem 3.1. Let $A=\left(a_{j n}\right)$ be non-negative regular summability matrix, the sequence $q=\left\{q_{n}\right\}$ satisfies (12) with $q_{n} \in(0,1]$ for all $n \in \mathbb{N}$. Then for every $f \in C_{\rho}^{0}[0, \infty)$, st $t_{A}-\lim _{n}\left\|L_{n, q_{n}}(f)-f\right\|_{\rho}=0$ where $\rho(x)=1+x^{2}$.

Proof. From Lemma1, it is obvious that $s t_{A}-\lim _{n}\left\|L_{n, q}\left(e_{0}\right)-e_{0}\right\|_{\rho}=0$. Using the (6), we get

$$
\begin{aligned}
\frac{\left|L_{n, q_{n}}\left(e_{1}\right)(x)-e_{1}(x)\right|}{1+x^{2}} & =\frac{x}{1+x^{2}}\left|\frac{D_{q_{n}} \varphi_{n}\left([n]_{q_{n}}^{\beta-1} x\right)}{[n]_{q_{n}} \varphi_{n}\left([n]_{q_{n}}^{\beta-1} x\right)}-1\right| \\
& \leq\left\|e_{1}\right\|_{\rho}\left|\frac{D_{q_{n}} \varphi_{n}\left([n]_{q_{n}}^{\beta-1} x\right)}{[n]_{q_{n}} \varphi_{n}\left([n]_{q_{n}}^{\beta-1} x\right)}-1\right| \\
& \leq\left|\frac{D_{q_{n}} \varphi_{n}\left([n]_{q_{n}}^{\beta-1} x\right)}{[n]_{q_{n}} \varphi_{n}\left([n]_{q_{n}}^{\beta-1} x\right)}-1\right|=B_{n, q_{n}}\left(\varphi_{n}, x\right)
\end{aligned}
$$

Now, for a given $\varepsilon>0$, we define the sets $U=\left\{n:\left\|L_{n, q_{n}}\left(e_{1}\right)-e_{1}\right\|_{\rho} \geq \varepsilon\right\}$ and $U_{1}=\left\{n: B_{n, q_{n}}\left(\varphi_{n}, x\right) \geq \varepsilon\right\}$. It is clear that $U \subset U_{1}$ and hence

$$
\delta_{A}\left\{n \in \mathbb{N}:\left\|L_{n, q_{n}}\left(e_{1}\right)-e_{1}\right\|_{\rho} \geq \varepsilon\right\} \leq \delta_{A}\left\{n \in \mathbb{N}: B_{n, q_{n}}\left(\varphi_{n}, x\right) \geq \varepsilon\right\} .
$$

From the condition (3) we get $s t_{A}-\lim B_{n, q_{n}}\left(\varphi_{n}, x\right)=0$. Therefore, it is clear that

$$
\delta_{A}\left\{n \in \mathbb{N}: B_{n, q_{n}}\left(\varphi_{n}, x\right) \geq \varepsilon\right\}=0
$$

and hence we have

$$
\delta_{A}\left\{n \in \mathbb{N}:\left\|L_{n, q_{n}}\left(e_{1}\right)-e_{1}\right\|_{\rho} \geq \varepsilon\right\}=0,
$$


which implies

$$
s t_{A}-\lim \left\|L_{n, q_{n}}\left(e_{1}\right)-e_{1}\right\|_{\rho}=0 .
$$

Similarly from (8) we can write

$$
\begin{aligned}
\left|L_{n, q_{n}}\left(e_{2}\right)-e_{2}\right|= & \left(\frac{D_{q_{n}}^{2} \varphi_{n}\left([n]_{q_{n}}^{\beta-1} x\right)}{q_{n}[n]_{q_{n}}^{2} \varphi_{n}\left([n]_{q_{n}}^{\beta-1} x\right)}-1\right) x^{2} \\
& +\frac{1}{[n]_{q_{n}}^{\beta}}\left(\frac{D_{q_{n}} \varphi_{n}\left([n]_{q_{n}}^{\beta-1} x\right)}{[n]_{q_{n}} \varphi_{n}\left([n]_{q_{n}}^{\beta-1} x\right)}-1\right) x+\frac{1}{[n]_{q_{n}}^{\beta}} x
\end{aligned}
$$

and hence we get

$$
\begin{aligned}
\frac{\left|L_{n, q_{n}}\left(e_{2}\right)-e_{2}\right|}{1+x^{2}} & \leq\left\|e_{2}\right\|_{\rho}\left|\frac{D_{q_{n}}^{2} \varphi_{n}\left([n]_{q_{n}}^{\beta-1} x\right)}{q_{n}[n]_{q_{n}}^{2} \varphi_{n}\left([n]_{q_{n}}^{\beta-1} x\right)}-1\right| \\
& +\frac{1}{[n]_{q_{n}}^{\beta}}\left\|e_{1}\right\|_{\rho}\left|\frac{D_{q_{n}} \varphi_{n}\left([n]_{q_{n}}^{\beta-1} x\right)}{[n]_{q_{n}} \varphi_{n}\left([n]_{q_{n}}^{\beta-1} x\right)}-1\right|+\frac{1}{[n]_{q_{n}}^{\beta}}\left\|e_{1}\right\|_{\rho} \\
& \leq B_{n, q_{n}}\left(\varphi_{n}, x\right)+\frac{1}{[n]_{q_{n}}^{\beta}} C_{n, q}\left(\varphi_{n}, x\right)+\frac{1}{[n]_{q_{n}}^{\beta}},
\end{aligned}
$$

Now for given $\varepsilon>0$, let us define the following sets

$$
\begin{aligned}
V & =\left\{n:\left\|L_{n, q_{n}}\left(e_{2}\right)-e_{2}\right\|_{\rho} \geq \varepsilon\right\}, \\
V_{1} & =\left\{n: B_{n, q_{n}}\left(\varphi_{n}, x\right) \geq \varepsilon / 3\right\}, \\
V_{2} & =\left\{n: C_{n, q_{n}}\left(\varphi_{n}, x\right) \geq \varepsilon / 3\right\}, \\
V_{3} & =\left\{n:[n]_{q_{n}}^{-\beta} \geq \varepsilon / 3\right\} .
\end{aligned}
$$

It is obviously that $V \subset V_{1} \cup V_{2} \cup V_{3}$. From the condition (12) we get

$$
s t_{A}-\lim \frac{1}{[n]_{q_{n}}^{\beta}}=s t_{A}-\lim _{n \rightarrow \infty}\left(\left(1-q_{n}\right) /\left(1-q_{n}^{n}\right)\right)^{\beta}=0
$$

with $0<\beta \leq 2 / 3$. Hence by the condition (3) we have $s t_{A}-\lim B_{n, q_{n}}\left(\varphi_{n}, x\right)=$ 0 and $s t_{A}-\lim C_{n, q_{n}}\left(\varphi_{n}, x\right)=0$. Then we obtain $\delta_{A}\left(V_{k}\right)=0, k=$ 1,2,3. Since $\delta_{A}(V) \leq \delta_{A}\left(V_{1}\right)+\delta_{A}\left(V_{2}\right)+\delta_{A}\left(V_{3}\right)$ we find that $s t_{A}-$ $\lim \left\|L_{n, q_{n}}\left(e_{2}\right)-e_{2}\right\|_{\rho}=0$.

Consequently we obtain that $s t_{A}-\lim \left\|L_{n, q_{n}}\left(e_{i}\right)-e_{i}\right\|_{\rho}=0, i=0,1,2$ which completes the proof of the Theorem according to the weighted Korovkin type Theorem $[12,6,10]$.

As a consequence, for all $n \in \mathbb{N}, x \geq 0$ and $0<q_{n}<1$, we have

$$
s t_{A}-\lim _{n}\left\|L_{n, q_{n}}\left(\left(e_{1}-e_{0} x\right)^{\nu}\right)\right\|_{\rho}=0, \nu=1,2
$$


where

$$
\begin{gathered}
L_{n, q_{n}}\left(\left(e_{1}-e_{0} x\right)\right)(x)=\left(\frac{D_{q_{n}} \varphi_{n}\left([n]_{q_{n}}^{\beta-1} x\right)}{[n]_{q_{n}} \varphi_{n}\left([n]_{q_{n}}^{\beta-1} x\right)}-1\right) x, \\
L_{n, q_{n}}\left(\left(e_{1}-e_{0} x\right)^{2}\right)(x) \\
=\left(\frac{D_{q_{n}}^{2} \varphi_{n}\left([n]_{q_{n}}^{\beta-1} x\right)}{q_{n}[n]_{q_{n}}^{2} \varphi_{n}\left([n]_{q_{n}}^{\beta-1} x\right)}-2 \frac{D_{q_{n}} \varphi_{n}\left([n]_{q_{n}}^{\beta-1} x\right)}{[n]_{q_{n}} \varphi_{n}\left([n]_{q_{n}}^{\beta-1} x\right)}+1\right) x^{2} \\
+\frac{1}{[n]_{q_{n}}^{\beta}}\left(\frac{D_{q_{n}} \varphi_{n}\left([n]_{q_{n}}^{\beta-1} x\right)}{[n]_{q_{n}} \varphi_{n}\left([n]_{q_{n}}^{\beta-1} x\right)}-1\right) x+\frac{1}{[n]_{q_{n}}^{\beta}} x .
\end{gathered}
$$

Theorem 3.2. Let $A=\left(a_{j n}\right)$ be non-negative regular summability matrix, the sequence $q=\left\{q_{n}\right\}$ satisfies (12) with $q_{n} \in(0,1]$ for all $n \in \mathbb{N}$. If any function $f \in C_{\rho}$, satisfies the Lipschitz condition that is

$$
|f(t)-f(x)| \leq M|t-x|^{\alpha}, 0 \leq \alpha<1, x, t \geq 0
$$

then

$$
s t_{A}-\limsup _{n} \frac{\left|L_{n, q_{n}}(f)(x)-f(x)\right|}{1+x^{\alpha}}=0
$$

where $M$ is a constant.

Proof. Since $L_{n, q_{n}}$ is a linear positive operator and $f$ satisfies the Lipschitz condition we can write,

$$
\begin{aligned}
& \left|L_{n, q_{n}}(f)(x)-f(x)\right| \leq L_{n, q_{n}}(|f(t)-f(x)|)(x) \\
\leq & \frac{M}{\varphi_{n}\left([n]_{q_{n}}^{\beta-1} x\right)} \sum_{k=0}^{\infty}\left|\frac{[k]_{q}}{[n]_{q_{n}}^{\beta} q_{n}^{k-1}}-x\right|^{\alpha} \frac{\left([n]_{q_{n}}^{\beta-1} x\right)_{n}^{k}}{[k]_{q_{n}} !} D_{q_{n}}^{k} \varphi_{n}(0) .
\end{aligned}
$$

Applying the Holder inequality with $p=2 / \alpha, s=2 /(2-\alpha)$ and saying $B_{n, q_{n}, k}\left(\varphi_{n} ; x\right):=\varphi_{n}^{-1}\left([n]_{q_{n}}^{\beta-1} x\right) \frac{\left([n]_{q_{n}}^{\beta-1} x\right)_{n}^{k}}{[k]_{q_{n}} !} D_{q_{n}}^{k} \varphi_{n}(0)$, from Lemma 1 we get

$$
\begin{aligned}
\left|L_{n, q_{n}}(f)(x)-f(x)\right| \leq & M\left(\sum_{k=0}^{\infty}\left(\frac{[k]_{q_{n}}}{[n]_{q_{n}}^{\beta} q_{n}^{k-1}}-x\right)^{2} B_{n, q_{n}, k}\left(\varphi_{n} ; x\right)\right)^{\alpha / 2} \\
& \times\left(\sum_{k=0}^{\infty} B_{n, q_{n}, k}\left(\varphi_{n} ; x\right)\right)^{(2-\alpha) / 2} \\
= & M\left(L_{n, q_{n}}\left(\left(e_{1}-e_{0} x\right)^{2}\right)(x)\right)^{\alpha / 2}
\end{aligned}
$$


Taking account to (16) and using the conditions (3) and (12),similarly with the proof of the Theorem1, we obtain the desired result.

Now, we concern with the order of approximation of a function $f \in C_{\rho}^{0}$ by the linear positive operator $L_{n, q}$. We will use the weighted modulus of continuity defined by

$$
\Omega_{m}(f ; \delta)=\sup _{x \in[0, \infty),|h| \leq \delta} \frac{|f(x+h)-f(x)|}{1+(x+h)^{m}},
$$

for each $f \in C_{\rho}^{0}, \rho(x)=1+x^{m}, x \in[0, \infty), m \in \mathbb{N}$.

The weighted modulus of continuity has the following properties (see [14]):

(i) $\lim _{\delta \rightarrow 0} \Omega_{m}(f ; \delta)=0$ for each $f \in C_{\rho}^{0}$

(ii) $\Omega_{m}(f ; \lambda \delta) \leq(\lambda+1) \Omega_{m}(f ; \delta)$ for each positive real number $\lambda, m \in \mathbb{N}$

(iii) $|f(t)-f(x)| \leq\left(1+(x+|t-x|)^{m}\right)\left(\frac{|t-x|}{\delta}+1\right) \Omega_{m}(f ; \delta)$ for every $x, t \in[0, \infty), m \in \mathbb{N}$.

Notice that, if $f$ is not uniformly continuous on the interval $[0, \infty)$; then the usual first modulus of continuity $\omega(f ; \delta)$ does not tend to zero, as $\delta \rightarrow 0$. It is seen that $\Omega_{m}(f ; \delta) \rightarrow 0$,as $\delta \rightarrow 0$ for all $f \in C_{\rho}^{0}$ due to the property (i).

We now give second our main result. The following theorem is given an estimate for the approximation error with the operators $L_{n, q_{n}}(f)$, by means of $\Omega_{1}(f ; \delta)$ with $\rho(x)=1+x$.

Theorem 3.3. Let $\left\{q_{n}\right\}$ be a sequence satisfying the condition (12) with $q_{n} \in(0,1]$ for all $n \in \mathbb{N}$. Suppose that the condition

$$
\left(\frac{D_{q_{n}}^{\nu} \varphi_{n}\left([n]_{q_{n}}^{\beta-1} x\right)}{q_{n}^{\nu-1}[n]_{q_{n}}^{\nu} \varphi_{n}\left([n]_{q_{n}}^{\beta-1} x\right)}-1\right)=O\left(1 /[n]_{q_{n}}^{\beta}\right)
$$

holds instead of (3). If $f \in C_{\rho}^{0}$ with $\rho(x)=1+x$ then the inequality

$$
\left\|L_{n, q_{n}}(f)(x)-f(x)\right\|_{\rho_{2}} \leq C \Omega_{1}\left(f ; 1 / \sqrt{[n]_{q_{n}}^{\beta}}\right)\left(1+1 /[n]_{q_{n}}^{\beta}\right)
$$

holds where $\rho_{2}(x)=1+x^{2}$ and $C$ is a constant independent of $f$ and $n$.

Proof. Considering the definition of $\Omega_{1}(f ; \delta)$ and by using the property (iii) of $\Omega_{1}(f ; \delta)$ we can write

$$
\begin{aligned}
|f(t)-f(x)| & \leq(1+x+|t-x|)\left(\frac{|t-x|}{\delta}+1\right) \Omega_{1}(f ; \delta) \\
& \leq(1+2 x+t)\left(\frac{|t-x|}{\delta}+1\right) \Omega_{1}(f ; \delta) .
\end{aligned}
$$


Since $L_{n, q_{n}}$ is a linear and positive operator we get

$$
\begin{aligned}
\left|L_{n, q_{n}}(f)(x)-f(x)\right| & \leq L_{n, q_{n}}(|f(t)-f(x)|)(x) \\
\leq & \Omega_{1}(f ; \delta)\left[L_{n, q_{n}}(1+2 x+t)(x)\right. \\
& \left.\quad+L_{n, q_{n}}\left((1+2 x+t) \frac{|t-x|}{\delta}\right)(x)\right] .
\end{aligned}
$$

To estimate the first term, considering (6) and (7), we can write

$$
\begin{aligned}
& \left(L_{n, q_{n}}((1+2 x+t))(x)\right) \\
& =(1+2 x) L_{n, q_{n}}\left(e_{0}\right)(x)+L_{n, q_{n}}\left(e_{1}\right)(x) \\
& =(1+3 x)+\left(\frac{1}{[n]_{q_{n}}^{\beta}} \frac{D_{q_{n}} \varphi_{n}\left([n]_{q_{n}}^{\beta-1} x\right)}{[n]_{q_{n}} \varphi_{n}\left([n]_{q_{n}}^{\beta-1} x\right)}-1\right) x \\
& \leq 3(1+x)\left[1+O\left(1 /[n]_{q_{n}}^{\beta}\right)\right] .
\end{aligned}
$$

Applying the Cauchy-Schwarz inequality to the second term in (18), since $L_{n, q_{n}}$ is a linear and positive, we get

$$
\begin{aligned}
& L_{n, q_{n}}\left((1+2 x+t) \frac{|t-x|}{\delta}\right)(x) \\
\leq & \left(L_{n, q_{n}}\left((1+2 x+t)^{2}\right)(x)\right)^{1 / 2} \times\left(L_{n, q_{n}}\left(\frac{(t-x)^{2}}{\delta^{2}}\right)(x)\right)^{1 / 2} .
\end{aligned}
$$

We now estimate the first term. By using (6),(7) and (8) and by simple calculations, we get

$$
\left(L_{n, q_{n}}\left((1+2 x+t)^{2}\right)(x)\right)^{1 / 2} \leq 4(1+x)\left[1+O\left(\frac{1}{[n]_{q_{n}}^{\beta}}\right)\right]^{1 / 2} .
$$

Taking into account (16), if we estimate the second term then we get

$$
\begin{aligned}
\left(L_{n, q_{n}}\left(\frac{|t-x|^{2}}{\delta^{2}}\right)(x)\right)^{1 / 2} & =\frac{1}{\delta}\left(L_{n, q_{n}}\left(\left(e_{1}-e_{0} x\right)^{2}\right)(x)\right)^{1 / 2} \\
& =\frac{1}{\delta}\left(O\left(\frac{1}{[n]_{q_{n}}^{\beta}}\right)\left(x^{2}+x\right)\right)^{1 / 2} \\
& \leq \frac{1}{\delta}\left(O\left(\frac{1}{[n]_{q_{n}}^{\beta}}\right)(1+x)^{2}\right)^{1 / 2} \\
& \leq \frac{1}{\delta}(1+x) \sqrt{\frac{C_{1}}{[n]_{q_{n}}^{\beta}}}
\end{aligned}
$$


with $C_{1}$ is a constant independent of $n$. Combining (19), (20), (21) and (22) with (18) we have

$$
\begin{aligned}
& \left|L_{n, q_{n}}(f)(x)-f(x)\right| \\
\leq & \Omega_{1}(f ; \delta) \times C_{2}(1+x)^{2}\left[1+O\left(1 /[n]_{q_{n}}^{\beta}\right)\right]\left(1+\frac{1}{\delta} \sqrt{\frac{1}{[n]_{q_{n}}^{\beta}}}\right),
\end{aligned}
$$

where $C_{2}=\max \left\{3,4 C_{1}\right\}$. Taking $\delta:=\delta_{n}=\left(1 /[n]_{q_{n}}^{\beta}\right)^{1 / 2}$ we obtain

$$
\begin{aligned}
\left|L_{n, q_{n}}(f)(x)-f(x)\right| & \leq 2 C_{2} \Omega_{1}\left(f ; \delta_{n}\right)\left(1+x^{2}\right)\left[C_{3}+O\left(1 /[n]_{q_{n}}^{\beta}\right)\right] \\
& \leq C \Omega_{1}\left(f ; \delta_{n}\right)\left(1+x^{2}\right)\left[1+O\left(1 /[n]_{q_{n}}^{\beta}\right)\right],
\end{aligned}
$$

with $C_{3}=\sup _{x \geq 0} \frac{(1+x)^{2}}{1+x^{2}}, C=\max \left\{2 C_{2}, C_{3}, C_{3} C_{1}\right\}$ which gives that the (17).

We notice that, from (13), it is clear that $s t_{A}-\lim _{n} \delta_{n} \rightarrow 0$ as $n \rightarrow \infty$. Therefore $s t_{A}-\lim _{n} \Omega_{1}\left(f ; \delta_{n}\right) \rightarrow 0$ due to the property (i) of $\Omega_{1}(f ; \delta)$. Consequently, order of $A$-statistical convergence of the sequence of $\left\{L_{n, q_{n}}(f)\right\}$ to $f$ is $\left(1 /[n]_{q_{n}}^{\beta}\right)^{1 / 2}$ in the $\rho_{2}$-norm.

\section{Local Approximation}

Theorem 4.1. Let $\left\{q_{n}\right\}$ be a sequence satisfying the condition (12) with $q_{n} \in(0,1]$ for all $n \in \mathbb{N}$. We have

1) For any $f \in C_{\rho}$ we have

$$
\left|L_{n, q_{n}}(f)(x)-f(x)\right| \leq 2 \omega\left(f ; \sqrt{\delta_{n, x}}\right)
$$

where $\omega(f, \delta)$ is the usual first modulus of continuity of $f$ and

$$
\delta_{n, x}=L_{n, q_{n}}\left(\left(e_{1}-e_{0} x\right)^{2}\right)(x)
$$

and $s t_{A}-\lim _{n} \delta_{n, x}=0$, for all fixed $x \in[0, \infty)$.

2) If $f \in C_{\rho}$ satisfies the Lipschitz condition then $\left|L_{n, q_{n}}(f)(x)-f(x)\right| \leq$ $M \delta_{n, x}^{\alpha / 2}, 0 \leq \alpha<1$.

Proof. 1) Using the linearity and positivity of the operator $L_{n, q_{n}}$ and the known properties of $\omega(f, \delta)$ and applying Cauchy -Schwarz inequality we obtain

$$
\begin{aligned}
& \left|L_{n, q_{n}}(f)(x)-f(x)\right| \leq L_{n, q_{n}}(|f(t)-f(x)|)(x) \\
\leq & \omega(f ; \delta)\left[L_{n, q_{n}}\left(e_{0}\right)(x)+\frac{1}{\delta}\left(L_{n, q_{n}}\left(e_{1}-e_{0} x\right)^{2}(x)\right)^{1 / 2}\right] .
\end{aligned}
$$

By choosing $\delta=\sqrt{\delta_{n, x}}$ as in (23), we reach the desired result. Notice that, taking into account (14), we get $s t_{A}-\lim _{n} \delta_{n, x}=0$ for all fixed $x$. 
Hence we have $s t_{A}-\lim _{n} \omega\left(f ; \delta_{n, x}\right)=0$. This gives the pointwise rate of $A$-statistical convergence of the operator $L_{n, q_{n}}(f)$ to the function $f$.

2) Considering the proof of Theorem 2 and formula (23) we obtain that

$$
\begin{aligned}
\left|L_{n, q_{n}}(f)(x)-f(x)\right| & \leq M\left(L_{n, q_{n}}\left(\left(e_{1}-e_{0} x\right)^{2}\right)(x)\right)^{\alpha / 2} \\
& =M \delta_{n, x}^{\alpha / 2} .
\end{aligned}
$$

Now we give the rate of $A$-statistical convergence for the operators $L_{n ; q_{n}}(f)$ by using the Peetre's $K$-functional in the space $C_{B}^{2}[0, \infty)$.

Let $C_{B}[0, \infty)$ be the space of all real valued uniformly continuous and bounded functions $f$ on the interval $[0, \infty)$ with the norm

$$
\|f\|_{C_{B}}=\sup _{x \in[0, \infty)}|f(x)| \text {. }
$$

The Peetre's $K$-functional of function $f \in C_{B}[0, \infty)$ is defined by

$$
\mathcal{K}(f ; \delta)=\inf _{g \in C_{B}^{2}}\left\{\|f-g\|_{C_{B}}+\delta\|g\|_{C_{B}^{2}}\right\}
$$

where $\delta>0$ and $C_{B}^{2}[0, \infty)=\left\{f \in C_{B}: f^{\prime}, f^{\prime \prime} \in C_{B}[0, \infty)\right\}$ endowed with the norm

$$
\|f\|_{C_{B}^{2}}=\|f\|_{C_{B}}+\left\|f^{\prime}\right\|_{C_{B}}+\left\|f^{\prime \prime}\right\|_{C_{B}} .
$$

Theorem 4.2. For each $f \in C_{B}^{2}[0, \infty)$ we have

$$
s t_{A}-\lim _{n}\left\|L_{n, q_{n}} f-f\right\|_{C_{B}}=0 .
$$

Proof. Applying the Taylor expansion to the function $f \in C_{B}^{2}[0, \infty)$, we can write

$$
\begin{aligned}
L_{n, q_{n}}(f)(x)-f(x)= & f^{\prime \prime}(x) L_{n, q_{n}}\left(\left(e_{1}-e_{0} x\right)\right)(x) \\
& +\frac{1}{2} f^{\prime \prime}(\zeta) L_{n, q_{n}}\left(\left(e_{1-} e_{0} x\right)^{2}\right)(x), \quad \zeta \in(t, x),
\end{aligned}
$$

where $L_{n, q_{n}}\left(\left(e_{1-} e_{0} x\right)\right)(x), L_{n, q_{n}}\left(\left(e_{1-} e_{0} x\right)^{2}\right)(x)$ are given by $(15)$ and (16) respectively.

Hence

$$
\begin{aligned}
\left\|L_{n, q_{n}}(f)-f\right\|_{C_{B}} \leq & \left\|f^{\prime}\right\|_{C_{B}}\left\|L_{n, q_{n}}\left(\left(e_{1}-e_{0} x\right)\right)\right\|_{C[0, A]} \\
& +\left\|f^{\prime \prime}\right\|_{C_{B}}\left\|L_{n, q_{n}}\left(\left(e_{1}-e_{0} x\right)^{2}\right)\right\|_{C[0, \alpha]} .
\end{aligned}
$$

Now for given $\varepsilon>0$, let us define $U=\left\{n \in \mathbb{N}:\left\|L_{n, q_{n}}(f)-f\right\|_{C[0, \alpha]} \geq \varepsilon\right\}$,

$$
U_{1}=\left\{n \in \mathbb{N}:\left\|f^{\prime}\right\|_{C_{B}}\left\|L_{n, q_{n}}\left(\left(e_{1}-e_{0} x\right)\right)\right\|_{C[0, \alpha]} \geq \varepsilon / 2\right\},
$$




$$
U_{2}=\left\{n \in \mathbb{N}:\left\|f^{\prime \prime}\right\|_{C_{B}}\left\|L_{n, q_{n}}\left(\left(e_{1}-e_{0} x\right)^{2}\right)\right\|_{C[0, \alpha]} \geq \varepsilon / 2\right\} .
$$

It is obvious that $U \subset U_{1} \cup U_{2}$ and hence $\delta_{A} U \leq \delta_{A} U_{1}+\delta_{A} U_{2}$. By using (14) we get $s t_{A}-\lim _{n}\left\|L_{n, q_{n}}\left(\left(e_{1}-e_{0} x\right)^{\nu}\right)\right\|_{C[0, \alpha]}=0, \nu=1,2$ with $[0, \alpha] \subset[0, \infty)$ and $\|,\|_{C[0, \alpha]}$ is maximum norm. Therefore we obtain $\delta_{A} U_{1}=0, \delta_{A} U_{2}=0$ so $\delta_{A} U=0$ which completes the proof.

Theorem 4.3. For each $f \in C_{B}[0, \infty)$

$$
\left\|L_{n, q_{n}}(f)-f\right\|_{C_{B}} \leq \mathcal{K}\left(f ; \delta_{n, x}\right)
$$

where $\left\{\mathcal{K}\left(f ; \delta_{n, x}\right)\right\}$ is the sequence of Peetre's $K$-functional and

$$
\delta_{n, x}=\left\|L_{n, q_{n}}\left(e_{1}-e_{0} x\right)\right\|_{C[0, \alpha]}+\left\|L_{n}\left(e_{1}-e_{0} x\right)^{2}\right\|_{C[0, \alpha]}
$$

and $s t_{A}-\lim _{n} \delta_{n, x}=0$ for each fixed $x \in[0, \infty)$.

Proof. For each $g \in C_{B}^{2}[0, \infty)$,by using (24) and ( 25), we get

$$
\begin{aligned}
\left\|L_{n, q} g-g\right\|_{C_{B}^{2}} \leq & \left(\left\|L_{n, q_{n}}\left(e_{1}-e_{0} x\right)\right\|_{C[0, \alpha]}\right. \\
& \left.+\left\|L_{n, q_{n}}\left(e_{1}-e_{0} x\right)^{2}\right\|_{C[0, \alpha]}\right)\|g\|_{C_{B}^{2}} \\
= & \delta_{n, x}\|g\|_{C_{B}^{2}} \text { say. }
\end{aligned}
$$

For each $f \in C_{B}[0, \infty)$ and $g \in C_{B}^{2}[0, \infty)$, we obtain

$$
\begin{aligned}
\left\|L_{n, q_{n}} f-f\right\|_{C_{B}^{2}} & \leq\left\|L_{n, q_{n}} f-L_{n, q_{n}} g\right\|_{C_{B}}+\left\|L_{n, q_{n}} g-g\right\|_{C_{B}^{2}}+\|g-f\|_{C_{B}} \\
& \leq 2\|g-f\|_{C_{B}}+\left\|L_{n, q_{n}} g-g\right\|_{C_{B}^{2}} \\
& \leq 2\|g-f\|_{C_{B}}+\delta_{n, x}\|g\|_{C_{B}^{2}} \\
& \leq 2\left(\|g-f\|_{C_{B}}+\delta_{n, x}\|g\|_{C_{B}^{2}}\right) .
\end{aligned}
$$

Taking the infimum on the right hand side over all $g \in C_{B}^{2}[0, \infty)$ we get

$$
\left\|L_{n, q_{n}} f-f\right\|_{C_{B}^{2}} \leq \mathcal{K}\left(f ; \delta_{n, x}\right) .
$$

By (14), we get $s t_{A}-\lim _{n} \delta_{n, x}=0$ so $s t_{A}-\lim _{n} \mathcal{K}\left(f ; \delta_{n, x}\right)=0$. Therefore we obtain the rate of $A$-statistical convergence of the sequence of the operators $L_{n, q_{n}}(f)$ to $f$ in the space $C_{B}[0, \infty)$.

\section{Concluding Remarks}

Some particular cases of the operators $L_{n, q}$ are defined as follows: 
a) If we take $\varphi_{n}(x)=(1+x)_{q}^{n}$ then we obtain $q$-Balazs-Szabados operators which are studied by O. Dogru [5]. In [5], the function $f$ has been taken as $f\left([k]_{q} /[n]_{q}^{\beta}\right)$ instead of $f\left([k]_{q} / q^{k-1}[n]_{q}^{\beta}\right)$ which is a natural generalization of $q$-Balazs-Szabados operators.

b) Taking into account the $q$-analogues of the exponential function given by $E_{q}\left([n]_{q} x\right)=\sum_{k=0}^{\infty} q^{k(k-1) / 2} \frac{\left([n]_{q}^{\beta} x\right)^{k}}{[k]_{q} !}$ and $e_{q}\left([n]_{q} x\right)=$ $\sum_{k=0}^{\infty} \frac{\left([n]_{q} x\right)^{k}}{[k]_{q} !}$ choosing $\varphi_{n}(x)=E_{q}\left(-[n]_{q} x\right)$ or $\varphi_{n}(x)=e_{q}\left(-[n]_{q} x\right)$, with $\beta=1$, we obtain $q$-Szasz-Mirakjan operators studied in different spaces in [1] and [20], respectively.

c) Taking $\varphi_{n}(x)=\left(1+q^{n-1} x\right)_{q}^{-n}$ we obtain the $q$-analogue of classical Baskakov operators studied in [2].

Consequently the $A$-statistical approximation properties are valid in large spectrum of the operators (5).

If we take $A=I$, identity matrix, we have the ordinary rate of convergence for the operators (5) (see, $[14,16]$ ).

\section{REFERENCES}

[1] A. Aral, A generalization of Szasz-Mirakyan operators based on q-integers, Math. Comput. Modelling. Vol. 47 (9-10),(2008), pp.1052-1062 .

[2] A. Aral,V. Gupta, On q-Baskakov type operators, Demonstratio Math., Vol. 42, No. 1, (2009),pp.109-122.

[3] K. Balazs, Approximation by Bernstein Type Rational Functions, Acta Mathematica Academiae Scientiarum Hungaricae Tomus,Vol. 26,No. 1-2, (1975),pp. 123-134.

[4] K. Balázs, J. Szabados, Approximation by Bernstein type rational function II, Acta Math. Acad. Sci. Hungar,Vol. 40,No. 3-4,(1982) pp.331-337.

[5] O. Dogru, On statistical approximation properties of Stancu type bivariate generalization of q-Balazs-Szabados operators, Proceedings of the Int. Conference on Numerical Analysis and Approximation Theory ,2006, Cluj-Napoca Romania,pp. 179-194.

[6] O. Duman, C. Orhan, Statistical approximation by positive linear operators, Stud. Math., Vol. 161, (2006),pp. 187-197.

[7] T. Ernst, The History of q-Calculus and a New Method U.U.D.M. Report 2000, Department of Mathematics, Uppsala University, Uppsala, 2000, p. 16.

[8] H. Fast, Sur la convergence statistique, Colloq. Math.,Vol. 2, (1951),pp.241-244.

[9] A. Fridy and H.L. Miller, A matrix characterization of statistical convergence, Analysis,Vol. 11,(1991),pp. 59-66 .

[10] Gadziev, A.D., The convergence problem for a sequence of positive linear operators on bounded sets and theorems analogous to that of P. P. Korovkin, Soviet Math. Dokl. , Vol. 15, No. 5, (1974). 
[11] A.D. Gadziev, On P.P. Korovkin type theorems, Zametki. Vol. 20, (in Russian), (1976),pp. 781-786 .

[12] A.D. Gadjiev and C. Orhan, Some Approximation theorems via statistical convergence, Rocky Mountain J. Math., Vol. 32,No. 1, (2002),pp.129-138 .

[13] V. Gupta and N. Ispir, On the Bezier variant of the generalized Kantorovich type Balazs operators, Appl. Math. Letters,Vol. 18 , (2005),pp.1053-1061.

[14] V. Gupta and N. Ispir, On the Kantorovich variant of generalized Bernstein type rational functions, Demons. Math.,Vol. 39, No. 1, (2006), pp. 117-130.

[15] V. Gupta and C. Radu, Statistical approximation properties of q-BaskokovKantorovich operators, Cent. Eur. J. Math., Vol. 7,No. 4 (2009),pp. 809-818.

[16] N. Ispir and C. Atakut, Approximation by generalized Balazs type rational functions, Int. J. Comput. Numer. Anal. Appl.,Vol. 4,No. 3, (2003), pp.297-316.

[17] Ispir N. and Gupta V., A-statistical approximation by the generalized KantorovichBernstein type rational operators,Southeast Asian Bull. Math.,Vol. 32,No. 1 (2008),pp. 87-97.

[18] N. Ispir, Rate of convergence of generalized rational type Baskakov operators, Math.Comput. Modelling, Vol. 46, (2007),pp. 625-631.

[19] E. Kolk, Statistically convergent sequences in normed spaces, Reports of convergence, Methods of algebra and analysis, Tartu,(1998), pp.63-66 .

[20] N. I. Mahmudov, Approximation properties of complex q-Szasz-Mirakjan operators in compact disks, Comput. Math. Appl., (2010), pp.1784-1791.

[21] Orkcu, M, Dogru, O., Weighted statistical approximation by Kantorovich type qSzasz-Mirakjan operators, Appl. Math. Comput., Vol. 217,No. 20, (2011),pp. 79137919 .

\author{
Nurhayat İsPir \\ Department of Mathematics \\ FACUlTy OF SCIENCES \\ GaZI UNIVERsity \\ 06500 ANKARA \\ TURKEY \\ E-mail address: nispir@gazi.edu.tr
}

